\title{
Update on Antithrombotic Therapy for Stroke Prevention in Atrial Fibrillation
}

\author{
Hermelinda G. Abcede, $M D^{*}$ \\ Bruce Ovbiagele, MD, MSC
}

\author{
Address \\ *Department of Neurology, University of California, Los Angeles, \\ 710 Westwood Plaza, Suite 1-240, Los Angeles, CA 90095, USA \\ Email: habcede@mednet.ucla.edu \\ Published online: 10 April 2010 \\ (C) The Author(s) 2010. This article is published with open access at Springerlink.com
}

\section{Opinion statement}

Atrial fibrillation (AF) is the most common cardiac arrhythmia in the elderly, affecting 1 in 20 adults over the age of 70 years. Stroke is a major yet highly preventable complication of AF, and the strokes related to AF often are disabling and fatal. Warfarin is the treatment of choice in high-risk patients with $A F$, and its superior efficacy over aspirin for preventing stroke in these patients is widely recognized. However, several eligible patients with AF are not being treated with warfarin or are being treated inadequately, largely because of concerns regarding the attendant strict monitoring, drug interactions, and risk of major bleeding. As such, alternative antithrombotic therapies that can rival or exceed the efficacy of warfarin, yet compare favorably with its administration and side effect profile, are being sought. One such strategy, the use of a combination antiplatelet regimen, for stroke prevention in high-risk patients with nonvalvular $\mathrm{AF}$ was investigated recently in two clinical trials. This article reviews the role of combination antiplatelet regimens in stroke prevention for patients with AF. Other therapies discussed include oral anticoagulation, single antiplatelet therapies, oral anticoagulation plus antiplatelet treatment, direct thrombin inhibitors, and factor Xa inhibitors.

\section{Introduction}

Atrial fibrillation (AF) is characterized by disordered electrical activity in the atria that causes an irregular and often rapid contraction of the ventricles [1]. AF may limit itself, recur (paroxysmal), or be persistent (lasting more than 7 days), and its overall prevalence increases with age, from $0.7 \%$ in persons aged 55 to 59 years to $18 \%$ in those 85 years and older [2]. The major complication of $\mathrm{AF}$ is systemic embolism (accounting for $\sim 50 \%$ of all cardiogenic emboli), mostly to the cerebral vascular bed, the latter of which manifests as strokes. After adjustment for other vascular risk factors, AF alone is associated with a three-to fourfold increased risk of stroke, and more than 75,000 cases of AF-related stroke are believed to occur each year in the United States [2]. These strokes generally are larger, more disabling, and more likely to be fatal than strokes of other causes.

Antithrombotic therapy is the cornerstone of stroke prevention among AF patients, and the incidence of 
ischemic stroke among patients with AF not treated with antithrombotic agents averages $4 \%$ to $5 \%$ per year, and may be greater than $13 \%$ per year in highrisk patients [3]. Oral anticoagulation, which generally involves the use of warfarin, currently is the treatment of choice for mitigating stroke risk in AF patients, but its use is limited by a narrow therapeutic index that demands strict monitoring, several drug and dietary interactions, a lack of firm caregiver commitment to ensure compliance with treatment and follow-up visits, and the risk of major bleeding, including hemorrhagic stroke [1]. Practitioners also have concerns about physical immobility from age-related health problems leading to falls and hemorrhagic complications, and whether participants in clinical trials, who generally are followed up more closely to ensure adherence to the study proto$\mathrm{col}$, are representative of patients seen in "real-world" practice, who may not necessarily be compliant with management protocols.

All the aforementioned factors have led to suboptimal warfarin use in clinical practice, with as many as $50 \%$ of eligible AF patients not receiving it, or up to three quarters not being treated adequately. As a result, various alternative antithrombotic therapies have, and continue to be, actively investigated for stroke prevention in patients with AF. One such regimen is combination antiplatelet therapy. This review article discusses up-to-date evidence-based antithrombotic treatment for stroke prevention in $\mathrm{AF}$ patients, with a major emphasis on the role of combination antiplatelet therapy.

\section{Antithrombotic treatment}

- Stroke in patients with AF is caused mainly by cardiogenic embolism. Over the years, various clinical trials of antiplatelet and anticoagulant medications to prevent stroke in AF have been conducted with the goal of interrupting the presumed cardioembolic mechanism of stroke in AF. In this section, the results of investigations of pertinent antithrombotic regimens are discussed.

\section{Single antiplatelet therapy (aspirin)}

- Aspirin prevents platelet activation by inhibiting the enzyme cyclooxygenase, thereby blocking thromboxane generation. A pooled analysis of individual patient-level data from three trials (Copenhagen Atrial Fibrillation, Aspirin and Anticoagulant Therapy Study [AFASAK] 1; Stroke Prevention in Atrial Fibrillation [SPAF] I; and European Atrial Fibrillation Trial [EAFT]) resulted in an estimated relative risk (RR) reduction of $21 \%$ for aspirin compared with placebo (95\% CI, 0\%-38\%) [4]. The confidence intervals for the pooled result indicate that the risk reduction across trials was barely significant. Other meta-analyses of aspirin versus control in AF patients have been conducted at the study level, not the patient level, and suggest a 22\% (95\% CI, 2\%-38\%) reduction in the risk of stroke in favor of aspirin [5].

- Pooled results from primary prevention trials of warfarin versus control have shown the superior efficacy of warfarin, which was consistent across studies, with an overall RR reduction of $68 \%(95 \%$ 


\section{Oral anticoagulation plus antiplatelet therapy}

- Because patients with AF often have coexisting atherosclerotic vascular disease, it is thought that both warfarin and aspirin may be necessary to simultaneously prevent thrombus formation in the left atrium and arteries [1]. Combining low-dose oral anticoagulation with aspirin appears to add relatively little protection against stroke compared with aspirin alone in patients with AF, whereas the combination of aspirin and oral anticoagulation at higher intensities significantly boosted the risk of intracranial hemorrhage, especially in elderly patients [10]. However, in the National Study for Prevention of Embolism in Atrial Fibrillation (NASPEAF), patients were stratified into a higher-risk group

\begin{tabular}{lcc}
\hline Table 1. Main implications of ACTIVE-A versus prior warfarin data (from meta-analyses) \\
\hline Effect & $\begin{array}{l}\text { Relative risk reduction, \% } \\
\text { Warfarin vs aspirin } \\
\text { (meta-analyses) [20] }\end{array}$ & $\begin{array}{c}\text { Clopidogrel plus aspirin vs } \\
\text { aspirin }\end{array}$ \\
& -38 & -28 \\
Reduction in stroke & +128 & +87 \\
Increase in intracranial hemorrhages & +70 & +51 \\
Increase in extracranial hemorrhages & & $+\bullet \cdot$ \\
ACTIVE-A-Atrial Fibrillation Clopidogrel Trial With Irbesartan for Prevention of Vascular Events & \\
(Adapted from Stuart J. Connolly, MD; with permission) &
\end{tabular}


(AF and rheumatic mitral stenosis or a history of embolism) and a lower-risk group (AF and age $>60$ years, hypertension, or heart failure) [11]. The higher-risk patients were randomly assigned to treatment with anticoagulation (INR 1.4-2.4) combined with the platelet cyclooxygenase inhibitor triflusal $(600 \mathrm{mg} / \mathrm{d}$, approximately equivalent to $300 \mathrm{mg}$ of aspirin) or anticoagulation (INR 2.0-3.0) alone. The lower-risk patients were randomly assigned to receive triflusal alone, anticoagulation (INR 2.0-3.0) alone, or triflusal plus anticoagulation to an INR of 1.25 to 2.0. The group receiving combination therapy had a significantly lower risk of primary outcome events (thromboembolism plus cardiovascular death) than the group treated with anticoagulation alone in both risk groups. Rates of severe bleeding, including intracerebral hemorrhage, were lower in the combination therapy arm than in the anticoagulation-only arm, but this difference was not statistically significant. However, it should be noted that the differences in primary outcome resulted largely from outcomes that probably were not a result of thromboembolism and that the achieved INR levels were similar in the anticoagulation and combination therapy groups.

- Currently, there is no evidence that combining anticoagulation with an antiplatelet agent reduces the risk of stroke or myocardial infarction compared with anticoagulant therapy alone in AF patients, but there is clear evidence of increased bleeding risk [10]. Therefore, in general, aspirin should not be added to anticoagulation therapy for AF patients without compelling indications for antiplatelet therapy.

- Presently there are few data regarding the efficacy of combining antiplatelet agents for stroke prevention in AF patients [12]. Available studies have examined the clinical impact of aspirin plus clopidogrel in AF patients. Clopidogrel is an adenosine diphosphate (ADP) receptor antagonist. Clopidogrel selectively and irreversibly inhibits ADP-induced fibrinogen binding to its receptor on platelets, thereby affecting ADP-dependent activation of the glycoprotein IIb/IIIa complex, the major receptor for fibrinogen present on the platelet surface. The value of combination antiplatelet therapy for stroke prevention in patients with AF was assessed in two ACTIVE (Atrial Fibrillation Clopidogrel Trial With Irbesartan for Prevention of

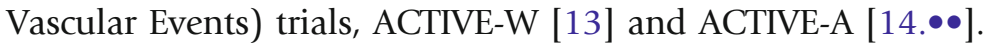

- ACTIVE-W evaluated the safety and efficacy of the combination of clopidogrel and aspirin versus warfarin in AF patients with at least one risk factor for stroke. This study was stopped prematurely by the safety monitoring committee after 3371 patients were enrolled because of the clear superiority of warfarin (INR 2.0-3.0) over the antiplatelet combination (RR, 1.44; 95\% CI, 1.18-1.76; $P=0.0003$ ). Random assignment to clopidogrel (75 mg once daily) plus aspirin 
$(75-100 \mathrm{mg} / \mathrm{d})$ was associated with a significant increase in the primary outcome of stroke, myocardial infarction, non-central nervous system (CNS) systemic embolism, or death from vascular causes after a median follow-up of 1.3 years compared with assignment to warfarin (target INR 2.0-3.0; 5.6\% vs 3.9\%; RR, 1.44; 95\% $\mathrm{CI}, 1.18-1.76)$. There was no significant difference in major bleeding between the two treatment groups (2.4\% per year for those on clopidogrel plus aspirin vs $2.2 \%$ per year for those on warfarin; RR, $1.10 ; 95 \%$ CI, 0.83-1.45). A subgroup analysis of the results of this trial raised the hypothesis that most of the benefit of warfarin over the combination of aspirin and clopidogrel was in patients who were already taking and tolerating oral anticoagulant therapy (ie, had survived the warfarin stress test), compared with patients who were warfarin naïve and about to start warfarin. Patients who were already taking warfarin at study entry and were randomly assigned to continue oral anticoagulation therapy had a substantial reduction in vascular events compared with those on the combination of clopidogrel and aspirin (3.7\% vs 5.5\% per year; RR, 0.67; 95\% CI, $0.55-0.84)$ and a significantly lower risk of major bleeding $(2.0 \%$ vs $2.6 \%$ per year; RR, 0.77 ; 95\% CI, 0.56-1.06). By contrast, patients who were not taking oral anticoagulants at study entry (ie, those who were warfarin naïve) and were randomly assigned to start oral anticoagulation had a similar rate of vascular events (4.7\% vs $5.9 \%$ per year; $\mathrm{RR}, 0.79 ; 95 \% \mathrm{CI}, 0.53-1.18)$ and a higher risk of major bleeding $(2.9 \%$ vs $1.7 \%$ per year; RR, 1.69 ; 95\% CI, 0.93-3.12) compared with those on the combination of clopidogrel and aspirin.

- ACTIVE-A followed an approach similar to that of ACTIVE-W but selected patients with AF for whom therapy with a vitamin K antagonist (VKA) was considered unsuitable. ACTIVE-A randomly assigned patients to receive clopidogrel plus aspirin or aspirin plus placebo. The reasons patients were not considered suitable for VKA therapy or enrollment in ACTIVE-W included the presence of a specific risk factor for bleeding (23\%), a physician assessment that the patient was not an appropriate candidate (50\%), and a patient preference not to receive a VKA (26\%). After a median follow-up of 3.6 years, compared with aspirin alone, clopidogrel once daily plus aspirin was associated with a reduction in the primary outcome of stroke, myocardial infarction, non-CNS systemic embolism, or death from vascular causes (6.8\% vs $7.6 \%$ per year; RR, 0.89 ; $95 \% \mathrm{CI}, 0.81-$ $0.98 ; P=0.01)$, mainly because of a reduction in the rate of stroke (2.4\% vs $3.3 \%$ per year; RR, 0.72; 95\% CI, 0.62-0.83; $P<0.001$ ) (Table 1, Table 2). The benefit of the combination in reducing major vascular events, however, was balanced by an increased risk of major hemorrhages (major vascular events decreased $0.8 \%$ per year, major hemorrhages increased $0.7 \%$ per year; RR, 0.97; 95\% CI, 0.89-1.06; $P=0.54)$. Major bleeding among patients assigned to receive clopidogrel plus aspirin versus those on aspirin alone was $2.0 \%$ versus 


\begin{tabular}{|c|c|c|c|c|c|c|c|c|}
\hline \multirow[b]{2}{*}{ Outcomes } & \multirow[b]{2}{*}{ Definition } & \multicolumn{2}{|c|}{$\begin{array}{l}\text { Treatment events } \\
\text { Clopidogrel plus } \\
\text { aspirin }\end{array}$} & \multicolumn{2}{|l|}{ Aspirin } & \multicolumn{3}{|c|}{$\begin{array}{l}\text { Clopidogrel plus aspirin } \\
\text { vs aspirin }\end{array}$} \\
\hline & & Number & Rate/year & Number & Rate/year & Relative risk & $95 \% \mathrm{CI}$ & $P$-value \\
\hline Primary & Composite & 832 & 6.8 & 924 & 7.6 & 0.89 & $0.81-0.98$ & 0.014 \\
\hline \multirow[t]{4}{*}{ Secondary } & Stroke & 296 & 2.4 & 408 & 3.3 & 0.72 & $0.62-0.83$ & $<0.001$ \\
\hline & Myocardial infarction & 90 & 0.7 & 115 & 0.9 & 0.78 & $0.59-1.03$ & 0.08 \\
\hline & Vascular death & 600 & 4.7 & 599 & 4.7 & 1.0 & $0.89-1.12$ & 0.97 \\
\hline & Non-CNS embolism & 54 & 0.4 & 56 & 0.4 & 0.96 & $0.66-1.40$ & 0.84 \\
\hline
\end{tabular}

$1.3 \%$ per year (RR, 1.57; 95\% CI, 1.29-1.92). Clopidogrel plus aspirin reduced the risk of stroke by $28 \%$ but increased the risk of major extracranial hemorrhage by 51\% (affecting predominantly the gastrointestinal tract) and major intracranial hemorrhage by $87 \%$ (Table 1).

- Ximelagatran, a direct thrombin inhibitor, was compared with doseadjusted warfarin in two major randomized controlled trials. In pooled analyses of both trials [10], the rate of main events (combined ischemic stroke, hemorrhagic stroke, and systemic embolic event) was similar in the ximelagatran and warfarin groups $(1.62 \%$ vs $1.65 \%$ per year). Conflated rates of minor and major bleeding were lower with ximelagatran ( $31.7 \%$ vs $38.7 \%$ per year; $P<0.0001$ ), but serum alanine aminotransferase values rose transiently to more than three times the normal level in the subjects on ximelagatran $(6.1 \%$ vs $0.8 \% ; P<0.0001)$. Although these increases usually are reversible, they led the US Food and Drug Administration (FDA) to decide against approving ximelagatran for stroke prevention.

- Dabigatran etexilate is a prodrug given orally in a fixed dose and quickly converted by cytochrome P-450-independent esterases to dabigatran, a powerful reversible direct competitive inhibitor of thrombin with a prompt onset of action, consistent anticoagulant effect, and half-life of 12 to $17 \mathrm{~h}$. RE-LY (Randomized Evaluation of Long-Term Anticoagulant Therapy), a prospective, open-label, randomized trial with blinded assessment of all outcomes, investigated

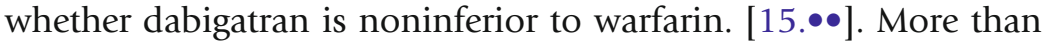
18,000 patients with nonvalvular AF and at least one risk factor for stroke were randomly assigned to receive fixed doses of dabigatran (110 or $150 \mathrm{mg}$ twice daily) or open-label, adjusted-dose warfarin (INR 2.0-3.0). After 2 years, the rates of systemic embolism or stroke 
were similar among patients who received $110 \mathrm{mg}$ dabigatran (1.53\% per year) and those who received warfarin (1.69\% per year; HR, 0.91; 95\% CI, 0.74-1.11; $P<0.001)$ and lower among patients who received dabigatran, $150 \mathrm{mg}$ twice daily $(1.11 \%$ per year; HR, $0.66 ; 95 \%$ CI, 0.53-0.82). Compared with warfarin (3.36\% per year), the yearly rate of major bleeding was lower among patients who received $110 \mathrm{mg}$ dabigatran $(2.71 \%$ per year; RR, 0.80; 95\% CI, 0.69-0.93) but similar among those assigned $150 \mathrm{mg}$ dabigatran (3.11\% per year; RR, 0.93; 95\% CI, 0.81-1.07). Compared with warfarin taken once daily, dabigatran is given twice daily, but the level of anticoagulation does not need to be monitored. Other drugs may interact with dabigatran, and its effects may not be reversed readily in patients with bleeding complications.

- Idraparinux is an analogue of the heparin pentasaccharide that binds irreversibly to antithrombin and stimulates a change leading to inactivation of activated factor X. It has a half-life of 80 to $130 \mathrm{~h}$ and is given by subcutaneous injection once weekly. Compared with warfarin, idraparinux has been associated with substantially higher bleeding rates [16.•]. A clinical trial that randomly assigned $4576 \mathrm{AF}$ patients to receive subcutaneous idraparinux $(2.5 \mathrm{mg} / \mathrm{wk})$ or adjusted-dose VKA therapy (target INR 2.0-3.0) had to be stopped after a mean follow-up of 11 months because of excess major bleeding among patients assigned to idraparinux versus VKA therapy (19.7 vs 11.3 events per 100 patient-years; HR, 1.74; 95\% CI, 1.472.06). Older patients and those with renal insufficiency were at greater risk for such complications. There was no difference in the rate of stroke and systemic embolism (0.9 vs 1.3 events per 100 patient-years; HR, $0.71 ; 95 \%$ CI, $0.39-1.30 ; P=0.007$ for noninferiority) or death (3.2 vs 2.9 deaths per 100 patient-years; $P=0.49$ ) between patients on idraparinux and those receiving VKA therapy. Several oral and subcutaneous factor Xa inhibitors currently are undergoing clinical evaluation.

\section{Current guidelines}

- Guidelines from the American Heart Association/American Stroke Association [3] and the American College of Chest Physicians [17.•] have not yet been updated to reflect the results of the ACTIVE-A and RE-LY trials but include the following:

- Initiate oral anticoagulation within 2 weeks of an ischemic stroke or transient ischemic attack (TIA); however, for patients with large infarcts, hemorrhagic transformation, or uncontrolled hypertension, further delays may be appropriate. 
- For patients with AF who suffer an ischemic stroke or TIA despite therapeutic anticoagulation, there are no data indicating that either increasing the intensity of anticoagulation or adding an antiplatelet agent provides additional protection against future ischemic events. In addition, both these strategies are associated with an increase in bleeding risk.

- Initiate warfarin for patients with AF and a moderate to high risk of stroke, such as those with a) mitral stenosis or a prosthetic heart valve, b) a history of prior ischemic stroke or systemic embolism, or c) two or more thromboembolic risk factors.

- In patients with AF, including those with paroxysmal AF, with only one major risk factor, initiate long-term antithrombotic therapy either as anticoagulation with an oral VKA, such as warfarin, targeted at an INR of 2.5 (range, 2.0-3.0), or as aspirin, at a dose of 75 to $325 \mathrm{mg} / \mathrm{d}$. For patients at intermediate risk for ischemic stroke, a VKA, rather than aspirin, is recommended.

- In patients with AF, including those with paroxysmal AF, aged 75 years or older with no major risk factors, initiate long-term aspirin therapy at a dose of 75 to $325 \mathrm{mg} / \mathrm{d}$ because of their low risk of ischemic stroke.

\section{FDA-approved treatments}

\section{Pharmacologic treatment}

- The current FDA-approved pharmacologic therapies for stroke prevention in patients with $\mathrm{AF}$ include dose-adjusted warfarin (targeted to INR 2.0-3.0) and antiplatelet agents, as discussed earlier [18, 19]. The only antiplatelet agents discussed in this section are aspirin and clopidogrel, as these are the two antiplatelets shown to reduce the rate of stroke in patients with AF.

\section{Antiplatelet agents}

Aspirin

Standard dosage 75 to $325 \mathrm{mg} / \mathrm{d}$ orally.

Contraindications Hypersensitivity to nonsteroidal anti-inflammatory drugs; children and teenagers with chickenpox or flu symptoms (risk of Reye's syndrome); syndrome of asthma, rhinitis, and nasal polyps.

Main drug interactions Ketorolac, citalopram, desvenlafaxine, dicumarol, duloxetine, eptifibatide, escitalopram, fluoxetine, fluvoxamine, ginkgo, heparin, ketoprofen, methotrexate, nefazodone, paroxetine, sertraline, ticlopidine, varicella virus vaccine, venlafaxine, warfarin.

Main side effects Gastrointestinal ulcers, bleeding, tinnitus, bronchospasm, angioedema, Reye's syndrome. 
Special points Patients should take aspirin with an 8-oz glass of water or food or milk. This drug should not be used in children because of the risk of Reye's syndrome. It should never be used in children and teenagers with chickenpox or flu symptoms.

Cost/cost-effectiveness 325-mg tablets (1 bottle, 100 each): \$11.99; 81-mg enteric coated tablets (1000 each): \$46.00: 975-mg enteric coated tablets (90 each): \$11.25; 325-mg enteric coated tablets (14 bottles, 100 each): $\$ 46.06$.

Clopidogrel

Standard dosage $75 \mathrm{mg} / \mathrm{d}$ orally.

Contraindications Active bleeding (eg, peptic ulcer or intracranial hemorrhage; hypersensitivity to clopidogrel.

Main drug interactions Abciximab, recombinant alteplase, argatroban, cilostazol, cimetidine, citalopram, dalteparin, desvenlafaxine, dicumarol, duloxetine, enoxaparin, eptifibatide, escitalopram, felbamate, fluconazole, fluoxetine, fluvoxamine, fondaparinux, heparin, ketoconazole, lansoprazole, nefazodone, omeprazole, pantoprazole, paroxetine, recombinant reteplase, sertraline, streptokinase, ticlopidine, urokinase, venlafaxine, voriconazole, warfarin.

Main side effects Chest pain, hypertension, pruritus, purpuric disorder, rash, hypercholesterolemia, abdominal pain, constipation, gastritis, indigestion, epistaxis, purpura, arthralgia, backache, headache.

Special points There have been reports of thrombotic thrombocytopenic purpura after exposure to clopidogrel.

Cost/cost-effectiveness 75-mg tablets (30 each): \$155.99.

\section{Oral anticoagulation}

\section{Warfarin}

Standard dosage

Contraindications

Main drug interactions
Initially, 2 to $5 \mathrm{mg} / \mathrm{d}$ orally; adjust dosage based on the INR.

Threatened abortion; eclampsia; preeclampsia; alcoholism; major regional or lumbar anesthesia; cerebral aneurysms; dissecting aortic aneurysms; bacterial endocarditis; bleeding tendencies of the gastrointestinal, genitourinary, or respiratory tract; blood dyscrasias; cerebrovascular hemorrhage; gastrointestinal, genitourinary, or respiratory tract ulcerations or overt bleeding; hypersensitivity to warfarin; inadequate laboratory facilities; malignant hypertension; pericarditis and pericardial effusion; pregnancy; lack of patient cooperation; spinal puncture and other procedures with potential for uncontrollable bleeding; recent or potential surgery of the CNS or eye; recent or potential traumatic surgery resulting in a large open surface.

Abciximab, recombinant alteplase, amiodarone, aspirin, carboplatin, celecoxib, chamomile, cilostazol, citalopram, clopidogrel, cyclophosphamide, dalteparin, desvenlafaxine, dicumarol, doxorubicin, duloxetine, enoxaparin, eptifibatide, escitalopram, etoposide, fenofibrate, fish oil, fluconazole, fluorouracil, fluoxetine, fluvoxamine, fondaparinux, garlic, ginkgo, heparin, influenza virus vaccine, ketoprofen, levofloxacin, marijuana, methotrexate, metronidazole, moxifloxacin, naproxen, papaya, paroxetine, pomegranate, 
Main side effects Cholesterol embolus syndrome, tissue necrosis, hemorrhage, hypersensitivity reaction (infrequent), intraocular hemorrhage.

Special points Warfarin may cause major or fatal bleeding. Risk factors for bleeding include a high intensity of anticoagulation (INR $>4.0$ ), age $\geq 65$ years, highly variable INRs, a

history of gastrointestinal bleeding, hypertension, cerebrovascular disease, serious heart disease, anemia, malignancy, trauma, renal insufficiency, concomitant drugs, and a long duration of warfarin therapy. Regular monitoring of INR is necessary. There are multiple significant drug-drug interactions. Patients should avoid alcohol, cranberry juice, and eating large amounts of food high in vitamin $\mathrm{K}$.

Cost/cost-effectiveness $\quad 1$-mg tablets (30 each): \$13.99; 2-mg tablets (30 each): \$14.88; 2.5-mg tablets (30 each): \$14.99; 3 -mg tablets (30 each): \$15.99; 4-mg tablets (30 each): \$14.99; 5-mg tablets (30 each): \$13.99; 7.5-mg tablets (30 each): \$23.21; 10-mg tablets (30 each): $\$ 24.24$.

\section{Acknowledgements}

Dr. Ovbiagele received support from the University of California, Los Angeles, Resource Centers for Minority Aging Research, Center for Health Improvement of Minority Elderly (RCMAR/CHIME) under National Institutes of Health/National Institute on Aging grant P30-AG021684.

\section{Disclosure}

No potential conflicts of interest relevant to this article were reported.

\section{Open Access}

This article is distributed under the terms of the Creative Commons Attribution Noncommercial License which permits any noncommercial use, distribution, and reproduction in any medium, provided the original author(s) and source are credited.

\section{References and Recommended Reading}

Papers of particular interest, published recently, have been highlighted as:

- Of importance

-. Of major importance

1. Lafuente-Lafuente C, Mahe I, Extramiana F: Management of atrial fibrillation. BMJ 2009, 339:b5216.

2. Wolf PA, Abbott RD, Kannel WB: Atrial fibrillation as an independent risk factor for stroke: the Framingham Study. Stroke 1991, 22:983-988.

3. Sacco RL, Adams R, Albers G, et al.: American Heart Association; American Stroke Association Council on Stroke; Council on Cardiovascular Radiology and Intervention; American Academy of Neurology: Guidelines for prevention of stroke in patients with ischemic stroke or transient ischemic attack: a 
statement for healthcare professionals from the American Heart Association/American Stroke Association Council on Stroke: co-sponsored by the Council on Cardiovascular Radiology and Intervention: the American Academy of Neurology affirms the value of this guideline. Stroke 2006, 37:577-617.

4. van Walraven C, Hart RG, Singer DE, et al.: Oral anticoagulants vs aspirin in nonvalvular atrial fibrillation: an individual patient meta-analysis. JAMA 2002, 288:2441-2448.

5. Hart RG, Benavente O, McBride R, Pearce LA: Antithrombotic therapy to prevent stroke in patients with atrial fibrillation: a meta-analysis. Ann Intern Med 1999, 131:492-501.

6. Halbmayer WM, Haushofer A, Schön R, Fischer M: The prevalence of poor anticoagulant response to activated protein C (APC resistance) among patients suffering from stroke or venous thrombosis and among healthy subjects. Blood Coagul Fibrinolysis 1994, 5:51-57.

7. Hylek EM, Skates SJ, Sheehan MA, et al.: An analysis of the lowest effective intensity of prophylactic anticoagulation for patients with nonrheumatic atrial fibrillation. $N$ Engl J Med 1996, 335:540-546.

8. Secondary prevention in non-rheumatic atrial fibrillation after transient ischaemic attack or minor stroke. EAFT (European Atrial Fibrillation Trial) Study Group. Lancet 1993, 342:1255-1262

9. Adjusted-dose warfarin versus low-intensity, fixed-dose warfarin plus aspirin for high-risk patients with atrial fibrillation: Stroke Prevention in Atrial Fibrillation III randomised clinical trial. Lancet 1996, 348:633-638

10. Akins PT, Feldman HA, Zoble RG, et al:: Secondary stroke prevention with ximelagatran versus warfarin in patients with atrial fibrillation: pooled analysis of SPORTIF III and V clinical trials. Stroke 2007, 38:874-880

11. Perez-Gomez F, Alegria E, Berjon J, et al.: Comparative effects of antiplatelet, anticoagulant, or combined therapy in patients with valvular and nonvalvular atrial fibrillation: a randomized multicenter study. $J$ Am Coll Cardiol 2004, 44(8):1557-1566.

12. Dale J, Myhre E, Storstein O, et al.: Prevention of arterial thromboembolism with acetylsalicylic acid. A controlled clinical study in patients with aortic ball valves. Am Heart J 1977, 94:101-111.

13. ACTIVE Writing Group of the ACTIVE Investigators, Connolly S, Pogue J, Hart R, et al.: Clopidogrel plus aspirin versus oral anticoagulation for atrial fibrillation in the Atrial fibrillation Clopidogrel Trial with Irbesartan for prevention of Vascular Events (ACTIVE W): a randomised controlled trial. Lancet 2006, 367:1903-1912.

$14 . \bullet$ ACTIVE Investigators, Connolly SJ, Pogue J, Hart RG, et al.: Effect of clopidogrel added to aspirin in patients with atrial fibrillation. N Engl J Med 2009, 360:2066-2078

This was the first randomized controlled trial to study the efficacy of combination antiplatelet therapy versus single antiplatelet treatment in nonvalvular AF patients who are not candidates for warfarin therapy. Dual antiplatelet therapy was more efficacious in preventing incident vascular events, particularly stroke, but at the price of significantly more major bleeding.

15.• Connolly SJ, Ezekowitz MD, Yusuf S, et al.: Dabigatran versus warfarin in patients with atrial fibrillation. N Engl J Med 2009, 361(12):1139-1151.

This randomized clinical trial evaluated the use of the direct thrombin inhibitor dabigatran compared with the gold standard, VKA therapy. Dabigatran appears to be a very promising alternative therapy to warfarin and may receive future regulatory approval for treatment of AF based on these results

16. Amadeus Investigators, Bousser MG, Bouthier J, Büller HR, Bousser MG, Bouthier J, Büller HR, et al.: Comparison of idraparinux with vitamin $\mathrm{K}$ antagonists for prevention of thromboembolism in patients with atrial fibrillation: a randomised, open-label, non-inferiority trial. Lancet 2008, 371(9609):315321.

This was the first major randomized trial to examine the role of a factor Xa inhibitor, idraparinux, versus the gold standard, VKAs. The trial was terminated early because of excess bleeding events in patients on idraparinux.

17. Singer DE, Albers GW, Dalen JE, et al.: American College of Chest Physicians: Antithrombotic therapy in atrial fibrillation: American College of Chest Physicians Evidence-Based Clinical Practice Guidelines (8th Edition). Chest 2008, 133:546S$592 S$.

These are the most recently published expert consensus guidelines on the appropriate use of antithrombotic therapy to prevent stroke in patients with $\mathrm{AF}$

18. Micromedex. Available at http://www.Thomsonhc. Com/home/dispatch. Accessed February 2010.

19. Epocrates. Available at http://www.Epocrates.com. Accessed February 2010.

20. Hart RG, Pearce LA, Aguilar MI: Meta-analysis: antithrombotic therapy to prevent stroke in patients who have nonvalvular atrial fibrillation. Ann Intern Med 2007, 146(12):857-867. 